\title{
Surface Concentration of Defects at Grain Boundaries in Sintered Alumina Determined by Positron Annihilation Lifetime Spectroscopy
}

\author{
J. KAnsY ${ }^{a}$, A. Si Ahmed ${ }^{b}$, J. Liebault ${ }^{b, c}$ AND G. MoYA ${ }^{b}$ \\ ${ }^{a}$ Institute of Physics and Chemistry of Metals, Silesian University \\ Bankowa 12, 40-007 Katowice, Poland \\ ${ }^{b}$ Laboratoire Matériaux et Microélectronique de Provence \\ (U.M.R./CNRS n ${ }^{0}$ 6137), Faculté des Sciences de St. Jérôme \\ Case 222, 13397 Marseille Cedex 20, France \\ ${ }^{c}$ Ecole Nationale Supérieure des Mines de Saint-Etienne \\ Département Céramiques Spéciales, 42023 Saint-Etienne Cedex 2, France
}

Sintered alumina samples of grain diameters spanning from 1.2 to $4.5 \mu \mathrm{m}$ have been investigated by positron annihilation lifetime spectroscopy. One series of samples was produced from material containing about $150 \mathrm{ppm}$ impurities (mainly $\mathrm{SiO}_{2}$ ). The second one was made from material having about $2700 \mathrm{ppm}$ of various elements $\left(\mathrm{SiO}_{2}, \mathrm{MgO}, \mathrm{CaO}\right)$. Two models of positron trapping at grain boundaries are compared: The first one relates to the diffusion-limited regime; and the other one - to the transition-limited regime of trapping. As a result the relative change of surface concentration of defects at grain boundaries is determined. Additionally, the positron diffusion constant in bulk alumina at room temperature, $D_{+}=0.36 \pm 10 \mathrm{~cm}^{2} / \mathrm{s}$, is estimated.

PACS numbers: 78.70.Bj, 77.84.Bw, 61.72.Mm

\section{Introduction}

It is well known that positron is a sensitive probe of imperfection in crystalline structure of solid states, e.g. [1]. In this work, we employ the positron annihilation lifetime spectroscopy to investigate the defect structure at grain boundaries of sintered alumina. Our interpretation of results is based on the diffusion-trapping 
model (DTM) proposed by Dupasquier et al. [2]. DTM describes the positron trapping in defects at grain boundaries taking into account the effects connected with finite positron diffusion in bulk material. For quick diffusion, i.e. when the diffusion length $L_{+}$is large, in comparison with the grain diameters, DTM reduces to the well-known two-states simple trapping model (STM). Therefore, the knowledge of $L_{+}$is essential for estimation of the distinction between both mentioned models.

The aim of this work is to measure $L_{+}$as well as the relative changes of $\sigma_{\mathrm{GB}}$, the surface concentration of defects at grain boundaries, as a function of the grain size and the content of impurities. The other objective is to estimate the influence of diffusion on the resulting values of $\sigma_{\mathrm{GB}}$. For this purpose, we put DTM into the code of our computer program. Additionally, in order to achieve maximal credibility of results, we collected the lifetime spectra with very high statistics reaching 20 millions of counts.

\section{Comparison between DTM and STM}

The diffusing trapping model makes use of the following assumptions [2]: (a) the material is formed by identical spherical grains of radius $R$, where annihilation occurs at a rate $\lambda_{\text {bulk }}$; (b) positron trapping occurs only at the surface of the domains (grain boundaries) of thickness $\delta \ll R$; (c) all trapped positrons annihilate with the same rate $\lambda_{\text {trap }}$; (d) the positrons are trapped in the grain boundaries only after their thermalization and the trapping rate is much lager than $\lambda_{\text {trap }}$; (e) the positron motion is governed by diffusion with a characteristic diffusion length $L_{+}$related to the positron diffusion constant $D_{+}$by the equation $L_{+}=\sqrt{D_{+} / \lambda_{\text {bulk }}}$.

The best distinction between the diffusion trapping and the transition trapping regimes gives the regime parameter $\alpha$ [2], which in our notation can be rewritten as

$$
\alpha=\frac{\nu}{\lambda_{\text {bulk }} R}\left(\frac{R}{L_{+}}\right)^{2} .
$$

The $\nu$ parameter describes the trapping efficiency and depends on the positron specific trapping rate $\mu\left(\mathrm{in}^{3} / \mathrm{s}\right)$ and the surface concentration of defects at grain boundaries $\sigma_{\mathrm{GB}}$

$$
\nu=\mu \sigma_{\mathrm{GB}}
$$

For $\alpha \gg 0$ the diffusion trapping occurs and the lifetime spectrum receives the form

$$
f(t)=\sum_{n=1, \infty} I_{n} \lambda_{n} \exp \left(-\lambda_{n} t\right)+I_{B} \lambda_{\text {trap }} \exp \left(-\lambda_{\text {trap }} t\right),
$$

where $I_{n}, I_{B}$, and $\lambda_{n}$ are known functions of $\lambda_{\text {bulk }}, \lambda_{\text {trap }}, L_{+}, R$, and $\nu$ of explicit form given in Ref. [2]. 
In the opposite case, for small $\alpha$, the trapping is mainly controlled by the transition rate and the STM description is sufficient. All the terms in the sum of Eq. (3), except the first one, disappear and $f(t)$ simplifies to two components.

\section{Experiment}

\subsection{Materials preparation}

The polycrystalline sintered alumina samples of our study were manufactured in a conventional laboratory environment from two types of powders (samples $\mathrm{E}$ and $\mathrm{B}$ ). The former (E), derived from the EXAL process, is considered for our purpose as pure ( $\sim 99.99 \%$ pure). The latter $(B)$, obtained through the BAYER process, contains about 2700 ppm of impurities ( $\sim 99.8 \%$ pure) and will be referred to as a doped material. The powder characteristics, according to the manufacturers, are presented in Table I.

TABLE I

Alumina powder characteristics as given by the manufactures.

\begin{tabular}{c|c|c|c|c|c|c|c}
\hline \hline \multirow{2}{*}{$\begin{array}{c}\text { Powder } \\
\text { type }\end{array}$} & $\begin{array}{c}\text { Manufacturer } \\
\text { (product } \\
\text { reference) }\end{array}$ & \multicolumn{4}{|c|}{ Impurities contents (ppm) } & Crystallite \\
\cline { 3 - 7 } & $\mathrm{SiO}_{2}$ & $\mathrm{CaO}$ & $\mathrm{MgO}$ & $\mathrm{Fe}_{2} \mathrm{O}_{3}$ & $\mathrm{Na}_{2} \mathrm{O}$ & $\begin{array}{c}\text { size } d_{50} \\
(\mu \mathrm{m})\end{array}$ \\
\hline $\mathrm{E}$ & $\begin{array}{c}\text { CRICERAM } \\
(\text { A67) }\end{array}$ & 90 & 5 & $<5$ & 12 & 40 & 0.5 \\
\hline $\mathrm{B}$ & $\begin{array}{c}\text { REYNOLDS } \\
\text { (RC172DBM) }\end{array}$ & 1497 & 686 & 723 & 415 & 404 & 0.4
\end{tabular}

TABLE II

Sintering temperatures, annealing times, and mean grain diameters of sintered samples.

\begin{tabular}{c|c|c|c|c|c}
\hline \hline \multicolumn{2}{l|}{ Samples } & $\begin{array}{c}\text { Sintering } \\
\text { temperature } \\
\left({ }^{\circ} \mathrm{C}\right)\end{array}$ & $\begin{array}{c}\text { Annealing time } \\
(\min )\end{array}$ & $\begin{array}{c}\text { Mean grain } \\
\text { diameter }(\mu \mathrm{m})\end{array}$ & $\begin{array}{c}\text { Relative } \\
\text { density } \\
(\%)\end{array}$ \\
\hline \multirow{5}{*}{ E } & E1 & 1590 & 100 & 1.7 & 98.7 \\
& $\mathrm{E} 2$ & 1620 & 120 & 2.5 & 98.8 \\
& $\mathrm{E} 3$ & 1650 & 120 & 4.5 & 99.1 \\
\hline \multirow{3}{*}{$\mathrm{B}$} & $\mathrm{B} 1$ & 1500 & 180 & 1.2 & 95.7 \\
& $\mathrm{~B} 2$ & 1550 & 180 & 2.0 & 98.0 \\
& B3 $^{*}$ & 1650 & 360 & 4.0 & 95.4 \\
\hline
\end{tabular}

${ }^{*}$ Precoarsening was achieved by a low-temperature soak $\left(1400^{\circ} \mathrm{C}\right.$ for $\left.4 \mathrm{~h}\right)$. 
The details of sintering processes have been fully described in previous work [3]. The sintering temperatures, the annealing times, and the resulting grains sizes as well as the densities are shown in Table II.

\subsection{Experimental set-up and data analysis}

The positron lifetime spectra were recorded at room temperature using a conventional fast-fast coincidence system with $\gamma$ detectors consisting of plastic scintillators characterised by a time resolution (FWHM) of 270 ps. The positron source, $10 \mu \mathrm{Ci}{ }^{22} \mathrm{Na}$ sealed by $0.75 \mu \mathrm{m}$ thick nickel foils, was sandwiched between two identical sintered pellets (of $20 \mathrm{~mm}$ diameter and $2 \mathrm{~mm}$ thickness). Each spectrum of the series was collected with a very high statistics which reaches 20 millions of counts.

The analysis was performed by means of LT program [4], with a new option being an implementation of the diffusion trapping model. Because the measured effect was very small $\left(I_{B}<5 \%\right)$ therefore a special calculation strategy had to be applied:

1. The resolution and the source correction parameters were fixed at mean values determined in a preliminary analysis. Similarly, according to the results of the preliminary analysis, the values of $\lambda_{\text {bulk }}$ and $\lambda_{\text {trap }}$ were established respectively as $1 / 137 \mathrm{ps}^{-1}$ and $1 / 283 \mathrm{ps}^{-1}$. The only parameters searched were $L_{+}$and $\nu$.

2. The resulting value of $L_{+}$(obtained in point 1 ) was used in the analysis of the whole series of spectra again. Thus the number of free parameters reduced to one, i.e. to $\nu$.

3. The procedure described in point 2 was repeated, but now $L_{+}$was chosen very large $(3000 \mathrm{~nm})$ to convert the theoretical model into STM.

\section{Results and discussion}

\subsection{Positron lifetimes in bulk and in defects}

The determined positrons lifetime in the bulk alumina, $\tau_{\text {bulk }}=\lambda_{\text {bulk }}^{-1}$, and the lifetime in the defects at grain boundaries, $\tau_{\text {trap }}=\tau_{\text {trap }}^{-1}$, are $\tau_{\text {bulk }}=137 \pm 0.1 \mathrm{ps}$ and $\tau_{\text {trap }}=183 \pm 23 \mathrm{ps}$, respectively. The first value is close to the lifetime of short-living component in single crystal and sintered alumina [5-7]. The second one can be compared with the lifetime in a vacancy in pure $\mathrm{Al}$ (250 ps) [8], or with the lifetimes in irradiation induced defects in single crystal of alumina (180-320 ps) [9].

\subsection{Positron diffusion in bulk alumina}

In spite of very high statistics of the experimental spectra they could be fitted by DTM as well as STM with nearly the same quality, i.e. the fit variances were almost independent on $L_{+}$. Only for one spectrum (of sample E3) an unambiguously result, $L_{+}=70 \pm 10 \mathrm{~nm}$, was obtained. Taking into account the grain 
radii $R$ (Table II), one can see that the condition $L_{+} \ll R$ is well satisfied for samples E3 $(R=2.25 \mu \mathrm{m})$ and for B3 $(R=2.0 \mu \mathrm{m})$. However, the respective values of $\alpha$ (Eq. (1)), calculated from the experimental parameters $L_{+}, \mu$, and $\lambda_{\text {bulk }}$, are about 11 and 1.8. The great dissimilarity between these values is caused mainly by different trapping efficiencies $\nu$, which are about 130 for E3 and about 30 for B3 (Fig. 1). The large value of $\alpha$ and high spectrum statistics ( 20 Mcounts), in the case of E3 sample, enabled us to distinguish between the DTM and STM approaches and to determine the $L_{+}$parameter.

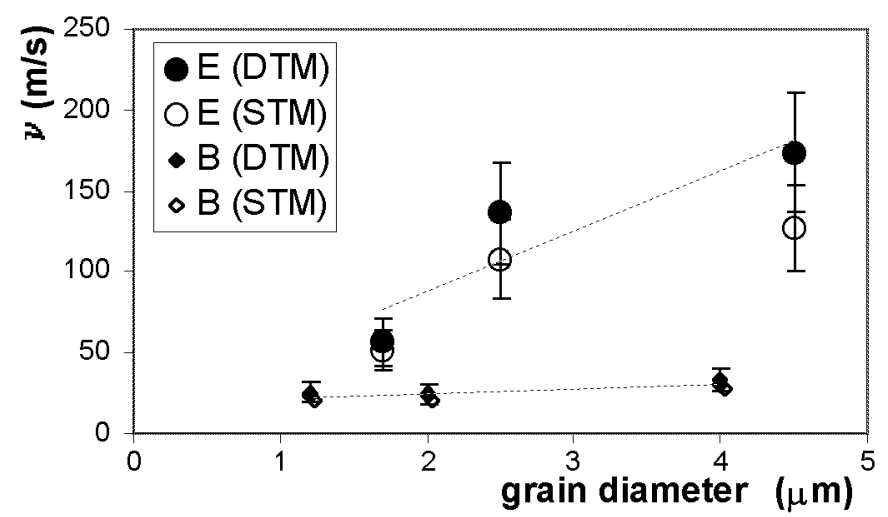

Fig. 1. The trapping efficiency versus the grain diameter for all the investigated spectra obtained with DTM and STM. Symbol E relates to "pure" samples, symbol B to the "doped" ones (Table I). The $\nu$ parameter reflects relative changes of $\sigma_{\mathrm{GB}}$, the surface concentration of defects at grain boundaries (Eq. (2)).

According to the assumption (e) in Sec. 2, the positron diffusion constant can be evaluated from the formula $D_{+}=L_{+}^{2} \lambda_{\text {bulk }}$. On the basis of our results, in bulk alumina at room temperature, $D_{+}$amounts to $0.36 \pm 10 \mathrm{~cm}^{2} / \mathrm{s}$.

\subsection{Surface concentration of defects at grain boundaries}

In Fig. 1, experimental values of $\nu$ (Eq. (2)) are given, for samples $\mathrm{E}$ and $\mathrm{B}$ (Table II), versus the grain diameters. For comparison, Fig. 1 shows the results of DTM and STM analyses. In reality, both analyses were carried out with the same numerical procedure but the latter one made use of the condition $L_{+} \gg R$, which reduced the diffusion model to the simple trapping model (see Sec. 3.2 point 3).

Providing that the specific trapping rate is the same for all samples then $\nu$ reflects changes in $\sigma_{\mathrm{GB}}$, the surface concentration of defects at grain boundaries (Eq. (2)). For the doped samples B (Table I), both DTM and STM give almost identical results. For pure samples E, the difference in $\sigma_{\mathrm{GB}}$, varies between $9 \%$ for E1 to $25 \%$ for E3. According to our preliminary interpretation: a) $\sigma_{\mathrm{GB}}$ illustrates 
the surface concentration of negatively charged vacancies (or small vacancy clusters) of Al mainly induced by Si atoms segregated at grain boundaries; b) the much higher $\sigma_{\mathrm{GB}}$, in the case of samples with a greater amount of impurities (B) than for "pure" samples (E), is caused by the formation of defect complex involving silicon and other foreign elements, which prevents Si from inducing the negatively charged Al vacancies.

The absolute values of $\sigma_{\mathrm{GB}}$ cannot by directly derived from Eq. (2), because an exact value of $\mu$ is unknown. Therefore, $\sigma_{\mathrm{GB}}$ can be only estimated by taking into account the probable order of $\mu$, i.e., $10^{14}-10^{15} \mathrm{ppm} / \mathrm{s}$ [8]. However $\mu$ in Eq. (2) is expressed by $\mathrm{m}^{3} / \mathrm{s}$. It is easy to see that $\mu\left[\mathrm{m}^{3} / \mathrm{s}\right]=\mu[\mathrm{ppm} / \mathrm{s}] \Omega / n_{A}$. Here $\Omega$ is the molecular volume and $n_{A}$ denotes the maximal number of defects, which can be generated in a molecule, so it is of the order of 1 . For alumina $\Omega=4.2 \times 10^{-29} \mathrm{~m}^{3}$. Taking into calculations $\mu=5 \times 10^{14} \mathrm{ppm} / \mathrm{s}$ one obtains $\mu=$ $2 \times 10^{-14} \mathrm{~m}^{3} / \mathrm{s}$. Finally, $\sigma_{\mathrm{GB}}$ can be estimated as $10^{15}$ defects $/ \mathrm{m}^{2}$, for samples B, and $(3-9) \times 10^{15}$ defects $/ \mathrm{m}^{2}$, for samples from E1 to E3.

\section{Conclusions}

1. The positron annihilation lifetime spectroscopy enables one to determine the positron diffusion constant at room temperature in grains of sintered alumina if the following conditions are satisfied: (a) the lifetime is collected with high statistics (of the order of 10 Mcounts), (b) the data analysis is carried out with DTM, (c) the grain diameters are about or greater than $4 \mu \mathrm{m}$ and (d) the trapping efficiency, $\nu$, amounts at least about $150 \mathrm{~m} / \mathrm{s}$.

2. For the investigated material, in room temperature, the diffusion trapping model can be substituted by the simple trapping model if $\nu<50 \mathrm{~m} / \mathrm{s}$. For $\nu$ above $100 \mathrm{~m} / \mathrm{s}$, the deviations between DTM and STM strongly depend on the grain size.

\section{References}

[1] Positron in Solids, Ed. P. Hautojärvi, Springer-Verlag, Berlin 1979.

[2] A. Dupasquier, R. Romero, A. Somoza, Phys. Rev. B 48, 9235 (1993).

[3] J. Liebault, J. Vallayer, D. Goeuriot, D. Treheux, F. Thevenot, J. Europ. Ceram. Soc. 21, 389 (2001)

[4] J. Kansy, Nucl. Instrum. Methods A 347, 235 (1996).

[5] M. Forster, W. Claudy, H. Hermes, M. Koch, K. Maier, J. Major, H. Stoll, H.E. Schaefer, Mater. Sci. Forum 105-110, 1005 (1992).

[6] H.E. Schaefer, M. Forster, Mater. Sci., Eng. A 109, 161 (1989).

[7] X.H. Li, D. Moya-Siesse, F. Salhi, G. Moya, Le Vide, Science, Technique et Applications 287, 494 (1998).

[8] H.E. Schaefer, Phys. Status Solidi A 102, 47 (1987).

[9] Y. Nagashima, K. Kawashima, T. Hyodo, M. Hasegawa, B.T. Lee, K. Hiraga, S. Yamaguchi, M. Forster, H.E. Schaefer, Mater. Sci. Forum 175-178, 461 (1995). 